\title{
Theory of Fiber Optic Lossless Polarizers
}

\author{
Stefan Wabnitz ${ }^{1}$, Victor V. Kozlov ${ }^{1,2}$, Javier Nuño ${ }^{3}$ \\ 1. University of Brescia, Department of Information Engineering, Via Branze 38, 25123, Brescia, Italy \\ 2. St.-Petersburg University, Department of Physics, St.-Petersburg, Petrodvoretz, 198504, Russia \\ 3. Instituto de Optica, Consejo Superior de Investigaciones Cientificas (CSIC), 28006 Madrid, Spain
}

We present a theoretical description of the light-by-light (nonlinear) polarization control of $\mathrm{CW}$ optical beams and short pulses. The present theory only deals with counter-propagating optical beams, for which the effects of group-velocity dispersion (GVD) are typically negligible. Indeed, GVD effects may only slightly modify the performance of lossless polarizers: therefore they are not significant for a basic understanding of the operation of these devices.

A lossless polarizer is a device which is able to polarize light with close to $100 \%$ efficiency. That is, any state of polarization (SOP) of a signal beam which is incident on the medium is transformed into one and the same SOP towards the output. The output SOP is set by the SOP of the counter-propagating pump beam. In contrast, passive (lossy) polarizers are characterized by an average $50 \%$ efficiency, and are non-conservative by nature. In essence, lossy polarizers do not transform the SOP of the incoming beam, but rather simply reject all polarization components that are not aligned with a particular polarization mode. Oftentimes, polarization-dependent losses which are inherent to passive polarizers may impair the operation of following optical devices. Therefore the main advantage of lossless polarizers is that they are conservative in nature, hence free of any polarizationdependent losses. Another advantage of lossless polarizers is that such devices enable a full light-by-light control of the SOP, in contrast with the need for an inconvenient and relatively slow mechanical control. These advantages, along with the simplicity of their practical implementation, make lossless polarizers (also called polarization funnels) attractive for polarization-selective optical communications and nonlinear photonics.

A lossless polarizer consists of a span of an optical fiber which is counter-pumped by a strong beam. The only fiber property which is known to be crucial for the performance of a lossless polarizer is its cubic nonlinearity, whereas the influence of the other effects is not known. The main goal of this study is the analysis of the effect that arise from nonzero birefringence: virtually all commercially available fibers exhibit some birefringence.

So far, the few available theoretical and experimental studies of lossless polarizers were mostly limited to the case of isotropic (non-birefringent) fibers, e.g. Ref. [1,2]. Truly isotropic fibers require a careful manufacturing: moreover, such fibers may typically preserve their isotropy for no longer than 1-2 meters of length. Such limitation entails high-power operation (of the order of $50 \mathrm{~W}$ ), which is necessary for introducing significant nonlinear phase shifts even with highly nonlinear fibers. Only very recently the disadvantages of isotropic fibers forced researchers to consider to real-world commercially available and low-cost telecommunication fibers. Indeed, the experimental study in Ref. [3] demonstrated an efficient lossless polarizer based on low-PMD telecommunication fibers (where PMD stands for the polarization-mode dispersion). Telecommunication fibers are birefringent, moreover their birefringence varies randomly along the fiber. Random birefringence strongly affects all polarization-related dynamics in the fiber, hence the very fact that a lossless polarizer could work in this case came as a surprise.

The main goal of this presentation is the description of the theory of lossless polarizers based on randomly birefringent fibers. Such theory explains why lossless polarizer may work in these of fibers, and also provides a good degree of predictability of new polarization effects. In addition to isotropic and randomly birefringent fibers, we also demonstrate that lossless polarizers may be based on highly-birefringent and unidirectionally spun highly nonlinear fibers [4]. As a matter of fact, we predict that in the last two cases the performance of the polarizer is typically better than what can be achieved with randomly birefringent fibers. As a culmination of our theory, we gather the models of all these fibers under a common umbrella and argue that the effect of polarization attraction, which is at the heart of lossless polarizers, is an intrinsic property of the boundary value problem for the underlying set of partial differential equations. These models may also pave the way for further analytical studies of the nonlinear dynamics of polarization interactions among different beams in optical fibers.

\section{References}

[1] S. Pitois, G. Millot, and S. Wabnitz, "Nonlinear polarization dynamics of couterpropagating waves in an isotropic optical fiber: theory and experiments," J. Opt. Soc. Am. B 18, 432-443 (2009).

[2] S. Pitois, J. Fatome, and G. Millot, "Polarization attraction using counterpropagating waves in optical fiber at telecommunication wavelengths," Opt. Express 16, 6646-6651 (2008).

[3] J. Fatome, S. Pitois, P. Morin, and G. Millot, " Observation of light-by-light polarization control and stabilization in optical fibre for telecommunication applications," Opt. Express 18, 15311-15317 (2010).

[4] V.V. Kozlov and S. Wabnitz, "Theoretical study of polarization attraction in high-birefringence and spun fibers," Opt. Lett. 35, 3949-3951 (2010). 